\title{
Reduction of Metabolic Cost during Motor Learning of Arm Reaching Dynamics
}

\author{
Helen J. Huang, Rodger Kram, and Alaa A. Ahmed \\ Department of Integrative Physiology, University of Colorado Boulder, Boulder, Colorado 80309-0354
}

It is often assumed that the CNS controls movements in a manner that minimizes energetic cost. While empirical evidence for actual metabolic minimization exists in locomotion, actual metabolic cost has yet to be measured during motor learning and/or arm reaching. Here, we measured metabolic power consumption using expired gas analysis, as humans learned novel arm reaching dynamics. We hypothesized that (1) metabolic power would decrease with motor learning and (2) muscle activity and coactivation would parallel changes in metabolic power. Seated subjects made horizontal planar reaching movements toward a target using a robotic arm. The novel dynamics involved compensating for a viscous curl force field that perturbed reaching movements. Metabolic power was measured continuously throughout the protocol. Subjects decreased movement error and learned the novel dynamics. By the end of learning, net metabolic power decreased by $\sim 20 \%(\sim 0.1 \mathrm{~W} / \mathrm{kg})$ from initial learning. Muscle activity and coactivation also decreased with motor learning. Interestingly, distinct and significant reductions in metabolic power occurred even after muscle activity and coactivation had stabilized and movement changes were small. These results provide the first evidence of actual metabolic reduction during motor learning and for a reaching task. Further, they suggest that muscle activity may not explain changes in metabolic cost as completely as previously thought. Additional mechanisms such as more subtle features of arm muscle activity, changes in activity of other muscles, and/or more efficient neural processes may also underlie the reduction in metabolic cost during motor learning.

\section{Introduction}

It is often assumed that the CNS controls movements in a manner that minimizes energetic cost. Indeed, using criteria that "minimize energy," mathematical models of movement can reproduce observed gait or arm reaching patterns (Nelson, 1983; Alexander, 1997; Kuo, 2001; Alexander, 2002; Todorov and Jordan, 2002; Emken et al., 2007; Franklin et al., 2008; Izawa et al., 2008). In these models, "energetic cost" is a generic term that can refer to a number of variables, including mechanical energy, motor command, effort, neural effort, muscle activity, or actual metabolic cost. If humans are truly minimizing energetic cost, then empirical studies should reveal that actual metabolic cost is minimized.

In locomotion, there are numerous experimental examples of minimizing actual metabolic cost. Humans walk and run using speeds (Ralston, 1958; Margaria, 1976), step lengths/frequencies (Cotes and Meade, 1960; Zarrugh et al., 1974; Cavanagh and Williams, 1982; Holt et al., 1991; Donelan et al., 2002), and step widths (Donelan et al., 2001; Arellano and Kram, 2011) that all correspond with the minimum metabolic cost. In general, walk-

\footnotetext{
Received Aug. 4, 2011; revised Dec. 9, 2011; accepted Dec. 14, 2011.

H.J.H., R.K., and A.A.A. designed research; H.J.H. performed research; H.J.H., R.K., and A.A.A. analyzed data; H.J.H., R.K., and A.A.A. wrote the paper.

This research was supported in part by NIH Grant 5T32AG000279 to H.J.H. Thanks to Bianca Bzdel and Andrew Kary for their help with data collections, and to members of the Neuromechanics Laboratory and Locomotion Laboratory for discussion about the project.

The authors declare no competing financial interests.

Correspondence should be addressed to Dr. Alaa A. Ahmed, Neuromechanics Laboratory, Department of Integrative Physiology, University of Colorado Boulder, 354 UCB, Boulder, C0 80309-0354. E-mail: alaa.ahmed@colorado.edu.

D0I:10.1523/JNEUROSCI.4003-11.2012

Copyright $\odot 2012$ the authors $\quad 0270-6474 / 12 / 322182-09 \$ 15.00 / 0$
}

ing or running with gait characteristics different from those preferred increases metabolic cost (Cotes and Meade, 1960; Zarrugh and Radcliffe, 1978; Donelan et al., 2001; Alexander, 2002; Arellano and Kram, 2011).

In contrast, only indirect evidence of metabolic minimization exists for motor learning or for arm reaching tasks. For example, when learning novel dynamics in an arm reaching task, subjects decrease muscle coactivation and stiffness (Thoroughman and Shadmehr, 1999; Franklin et al., 2003; Darainy and Ostry, 2008). This learning process involves forming and updating an internal model, a sensorimotor map of the system's dynamics, which the nervous system uses to predict movement dynamics and generate anticipatory forces (Shadmehr and Mussa-Ivaldi, 1994). As the internal model is learned, subjects can exert the specific muscle forces or joint torques needed to counteract any perturbing forces, and thereby, decrease muscle coactivation and "wasted energy" (Thoroughman and Shadmehr, 1999). Because motor learning and arm reaching studies to date have not included actual measures of metabolic cost (i.e., via expired gas analysis), we do not know whether actual metabolic cost is truly minimized, or even reduced, during motor learning and/or arm reaching.

The goal of this study was to measure actual metabolic power consumption using expired gas analysis as subjects learned novel arm reaching dynamics. We use "metabolic cost" to refer to a generic cost, and "metabolic power" to refer to our empirical measures of metabolic cost. We used the well studied motor-learning paradigm of reaching in a viscous curl force field using a robotic arm (Shadmehr and Mussa-Ivaldi, 1994). We hypothesized that metabolic power output would decrease as the novel dynamics were learned. This would support 

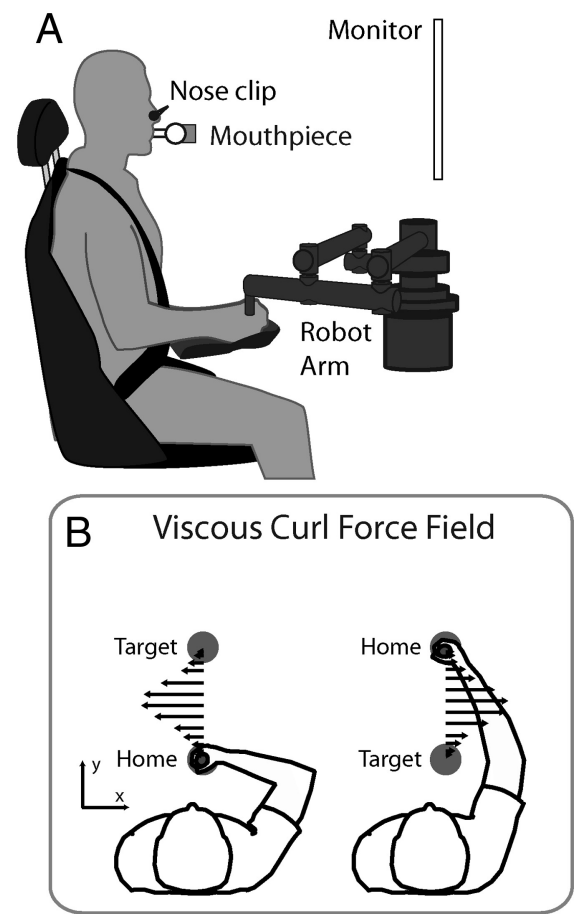

Figure 1. Experiment setup and force fields: $\boldsymbol{A}$, Subjects made horizontal planar reaching movements using a robotic arm, while breathing through a mouthpiece to measure rates of oxygen consumption and carbon dioxide production. The subject's arm was supported in a cradle attached to the robot handle. Odd numbered trials involved reaching outwards to the target while even trials involved reaching inwards. An auditory metronome paced subjects to start movements at 2 sintervals. $\boldsymbol{B}$, Schematic of the viscous curl force field. On outward movements, the force field applied a perturbation to the left $(-x)$ and for inward movements, the perturbation was to the right $(+x)$. $C$, Schematic of the force channel used during the catch trials to measure the anticipatory force subjects planned to use to counter the perturbing force of the curl force field.

the concept that the CNS reduces metabolic cost during movement. Our second hypothesis was that muscle activity and coactivation would parallel the decrease in metabolic power, based on studies that suggest that decreased muscle coactivation implies a decrease in metabolic cost (Thoroughman and Shadmehr, 1999; Franklin et al., 2004).

\section{Materials and Methods}

Subjects. Fifteen right-handed subjects (age $23.8 \pm 4.7$ years, mass $66.9 \pm$ $12.6 \mathrm{~kg}, 13$ females, 2 males) participated in this study. All subjects were healthy with no physical injuries or known pathologies. Subjects gave informed consent in accordance with the University of Colorado's Institutional Review Board.

Movement task. Subjects sat in a chair with full back support and made horizontal planar reaching movements while grasping the handle of a robotic arm (Interactive Motion Technologies, Shoulder-Elbow Robot 2; Fig. $1 \mathrm{~A}$ ). The task was to move a cursor (representing the handle position) from a home circle to a target circle $20 \mathrm{~cm}$ away. The cursor, home circle, and target circle were displayed on a computer monitor suspended vertically in front of the subject at eye-level. The target and home circles switched positions such that trials alternated between outward and inward movements. Visual feedback encouraged subjects to reach the target and complete movements within $300-600 \mathrm{~ms}$, while an auditory metronome paced subjects to initiate movements every $2 \mathrm{~s}$. Targets were within arm's reach of the subjects and did not require trunk movement. Bilateral shoulder straps and a lap belt limited torso movement.

Robot generated force fields. We used a viscous curl force field (Eq. 1) to add novel dynamics to the arm reaching task, where $b=-20 \mathrm{~N} \cdot \mathrm{s} / \mathrm{m}$.

$$
\left[\begin{array}{l}
F_{x} \\
F_{y}
\end{array}\right]=b\left[\begin{array}{rr}
0 & 1 \\
-1 & 0
\end{array}\right]\left[\begin{array}{l}
V_{x} \\
V_{y}
\end{array}\right] .
$$

This curl force field produced a perturbing force, $F$, that was perpendicular and proportional to handle velocity, $V$, (Fig. $1 B$ ). In addition, one in every five trials was randomly designated as a catch trial, which applied a force "channel" that simulated stiff walls along the straight path between the home and target circles (Fig. 1C). Catch trials allowed us to measure anticipatory force, defined as the force that subjects applied into the wall of the channel that would have counteracted the perturbing force. Anticipatory force was a measure of how well subjects had learned the dynamics of the curl force field and the accuracy of their internal model learning.

Experimental protocol. The experiment was organized into six blocks (Fig. 2). The experiment began with 10 min of quiet sitting to establish a baseline resting metabolic power. The 10 min period allowed subjects time to become comfortable with breathing through the mouthpiece and allowed subjects to settle to a steady-state resting metabolic rate. Subjects then performed 200 null trials of reaching to the target with no force field (Null 1). Next, the curl force field was engaged and subjects performed another 250 trials (Force 1). Subjects were given a brief rest, $\sim 3 \mathrm{~min}$, during which they did not have to breathe through the mouthpiece. Upon resuming the protocol, subjects completed another 250 trials in the curl force field (Force 2), followed by another 200 null trials to washout the learning (Null 2). The same curl force field was used in the Force 1 and Force 2 blocks. The experiment concluded with 10 min of quiet sitting.

Movement error and anticipatory force (metrics of motor learning). The handle position, handle velocity, and robot generated force were recorded at $200 \mathrm{~Hz}$. Movement error during a trial was defined as the maximum magnitude of the perpendicular deviation of the handle from a straight line path between the home and target circles. We refer to this movement deviation as movement error, even though subjects were not explicitly instructed to move in a straight line. Because reductions in movement error can occur with increased muscle coactivation and/or joint stiffness, reduced movement error does not necessarily indicate learning of the dynamics. Anticipatory force is a measure of internal model learning and was quantified as the maximum magnitude of the force exerted into the walls of the channel during a catch trial.

Metabolic power. Subjects wore a nose clip and breathed in and out of a mouthpiece throughout the protocol so that we could measure their rates of $\mathrm{O}_{2}$ consumption $\left(\dot{\mathrm{VO}}_{2}\right)$ and $\mathrm{CO}_{2}$ production $\left(\dot{\mathrm{V} C \mathrm{O}_{2}}\right)$ using expired gas analysis (ParvoMedics, TrueMax2400). The metabolic system was calibrated before each data collection using certified gas mixtures and with a range of flow rates using a $3 \mathrm{~L}$ calibration syringe. The metabolic system corrected all data with respect to standard temperature and pressure, dry, and averaged data in $\sim 15 \mathrm{~s}$ time intervals. All subjects had respiratory exchange ratios $\left(\mathrm{RER}=\dot{\mathrm{V} C \mathrm{C}_{2}} / \mathrm{V}_{2}\right)<1$ during the experimental protocol, which indicated that predominantly aerobic metabolism was involved.

Using $\dot{\mathrm{VO}}_{2}$ and $\dot{\mathrm{V}} \mathrm{CO}_{2}$, we calculated metabolic power in watts using the Brockway equation (Brockway, 1987). We also normalized by body mass to obtain metabolic power in units of $\mathrm{W} / \mathrm{kg}$. The metabolic measuring system averaged data for an integral number of breaths, so for consistency, we computed the time-weighted mean of the metabolic power for the last $2 \mathrm{~min}$ of each block. We used the last $2 \mathrm{~min}$ of each block to compare steady-state metabolic power for steady-state movement patterns. Averaging during the last $1-3$ min of a task is the standard approach for analyzing metabolic data (Brooks et al., 1996; Donelan et 


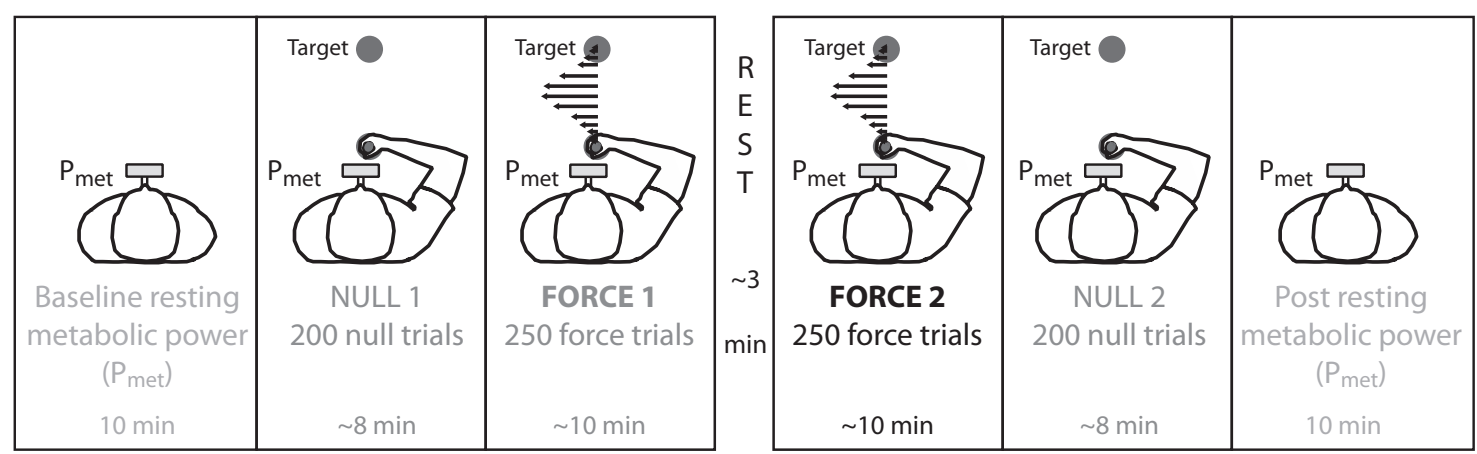

Data hierarchy example

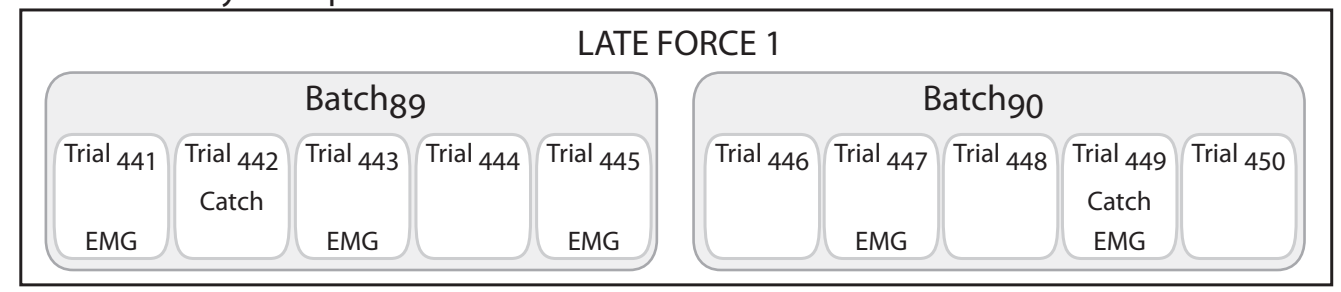

Figure 2. Experimental protocol and example of data hierarchy. There were 6 blocks: baseline resting (light gray), Null 1 (gray), Force 1 (bold gray), Force 2 (bold black), Null 2 (gray), and post resting (light gray). Colors are used in other figures to associate data with specific blocks. During the Force 1 and Force 2 blocks, subjects made reaching movements in the curl force field. All metrics were calculated early and late in each reaching block (Null 1, Force 1, Force 2, and Null 2). $P_{\text {met, }}$ metabolic power. Trial attributes for late Force 1 are provided as an example of the data hierarchy. A batch consisted of five trials. One trial within each batch was a catch trial. Electromyographic data (EMG) were collected for every odd numbered trial.

al., 2001; Gottschall and Kram, 2003; Grabowski et al., 2005; Houdijk et al., 2009; Arellano and Kram, 2011; Farris and Sawicki, 2011; Snyder and Farley, 2011). We also estimated metabolic power early within a block by averaging metabolic power for minutes $\sim 2-4$ of the block. We did not include the first $\sim 2$ min of the block to account for time delays in the measurement system. We calculated the net metabolic power by subtracting the baseline resting metabolic power from the metabolic power data during the reaching blocks (Null 1, Force 1, Force 2, and Null 2).

Electromyography and muscle coactivation. In 7 subjects, we also collected surface electromyographic (EMG) data (Delsys Trigno) from six upper limb muscles: pectoralis major, posterior deltoid, biceps brachii, triceps long head, triceps lateral head, and the brachioradialis. These arm muscles are the predominant muscles used during force field reaching (Thoroughman and Shadmehr, 1999; Franklin et al., 2003; Darainy and Ostry, 2008; Franklin et al., 2008). We placed electrodes according to published guidelines (www.seniam.org; Cram and Kasman, 1998). For each muscle belly surface, we shaved and cleaned the skin area with alcohol. The EMG data were sampled at $2000 \mathrm{~Hz}$ and hardware bandpass filtered $(20-450 \mathrm{~Hz})$. We used a signal sent out from the robot system to trigger the start and stop of each EMG recording for a trial. Because the electromyography system required time to reset before it could be triggered again and because we wanted subjects to initiate movements every $2 \mathrm{~s}$, we only collected EMG data for every other trial (i.e., odd trials, outward movements). To smooth the EMG data, signals were digitally high-pass filtered using a fourth order zero-lag Butterworth filter (MathWorks, Inc., MATLAB) with a cutoff of $20 \mathrm{~Hz}$, full wave rectified, and then low-pass filtered with a cutoff of $50 \mathrm{~Hz}$.

To normalize the EMG data, we calculated the root-mean-square (RMS) amplitudes for each muscle for the last 25 noncatch EMG trials in Null 1 and then used the maximum RMS among these trials as the normalization value. We used this task-based normalization method instead of a maximum voluntary contraction-based normalization method to reduce intersubject variability (Yang and Winter, 1984; Burden, 2010).

For each muscle, we quantified the RMS amplitude of the normalized electromyogram (RMS EMG) for the time after the cursor left the home circle until the time the cursor reached the target circle in each EMG trial. We also calculated RMS coactivation amplitudes for three muscle pairs: pectoralis major-posterior deltoid pair, biceps brachii-triceps long head pair, and brachioradialis-triceps lateral head pair. For each time point of the EMG data, we identified the minimum normalized EMG activity level of the muscle pair to obtain a coactivation profile for the EMG trial. This coactivation profile represented the "wasted contraction" (Thoroughman and Shadmehr, 1999; Gribble et al., 2003). We then calculated the RMS of the coactivation profile to get a RMS coactivation per EMG trial. For this first study, we analyzed muscle activity amplitudes and coactivation amplitudes, similar to previous force field reaching studies that measured surface electromyography (Thoroughman and Shadmehr, 1999; Franklin et al., 2003, 2008).

Time course analysis using batches. To examine the time course of the motor learning metrics and of the metabolic power during the experiment and across subjects, we analyzed data in batches of 5 trials (900 trials $/ 5$ trials $=180$ batches). This was necessary because we wanted to align the metabolic data, which were recorded approximately every $15 \mathrm{~s}$, with respect to motor learning trial data, which occurred approximately every $2 \mathrm{~s}$. By grouping trials into batches of 5 trials, we could average the trial data for each batch, yielding batch data with time periods of $10-12 \mathrm{~s}$, which were more similar to the $\sim 15 \mathrm{~s}$ time periods for the metabolic data. To calculate the metabolic power during each batch, we interpolated the metabolic data at the average time for each batch. Within each batch, one trial was a catch trial that was excluded when calculating the average movement error for each batch (i.e., average of the 4 noncatch trials). The anticipatory force for each batch was the value of the anticipatory force in the catch trial of that batch. Because we only collected EMG data for every odd numbered trial, we analyzed RMS EMG and RMS coactivation data in groups of $5 \mathrm{EMG}$ trials.

Overall motor learning comparisons. To quantify changes of overall motor learning, we compared all metrics at the following time points of the protocol: early Null 1, late Null 1 (baseline), early Force 1, late Force 1, early Force 2, late Force 2, early Null 2, and late Null 2. For movement error, "early" consisted of the first trial in the block, whereas "late" consisted of the average of the last 8 noncatch trials. For anticipatory force, "early" consisted of the first catch trial in the block, whereas "late" consisted of the average of the last 2 catch trials. For net metabolic power, "early" was estimated using the net metabolic power for minutes $\sim 2-4$ of the block whereas "late" was the net metabolic power during the last 2 min of the block. Last, for RMS EMG and RMS coactivation, "early" was the first 10 noncatch EMG trials, whereas "late" was the last 25 noncatch EMG trials in the block. More EMG trials were included in early and late time points because of greater trial-to-trial variability in RMS EMG and RMS coactivation. 

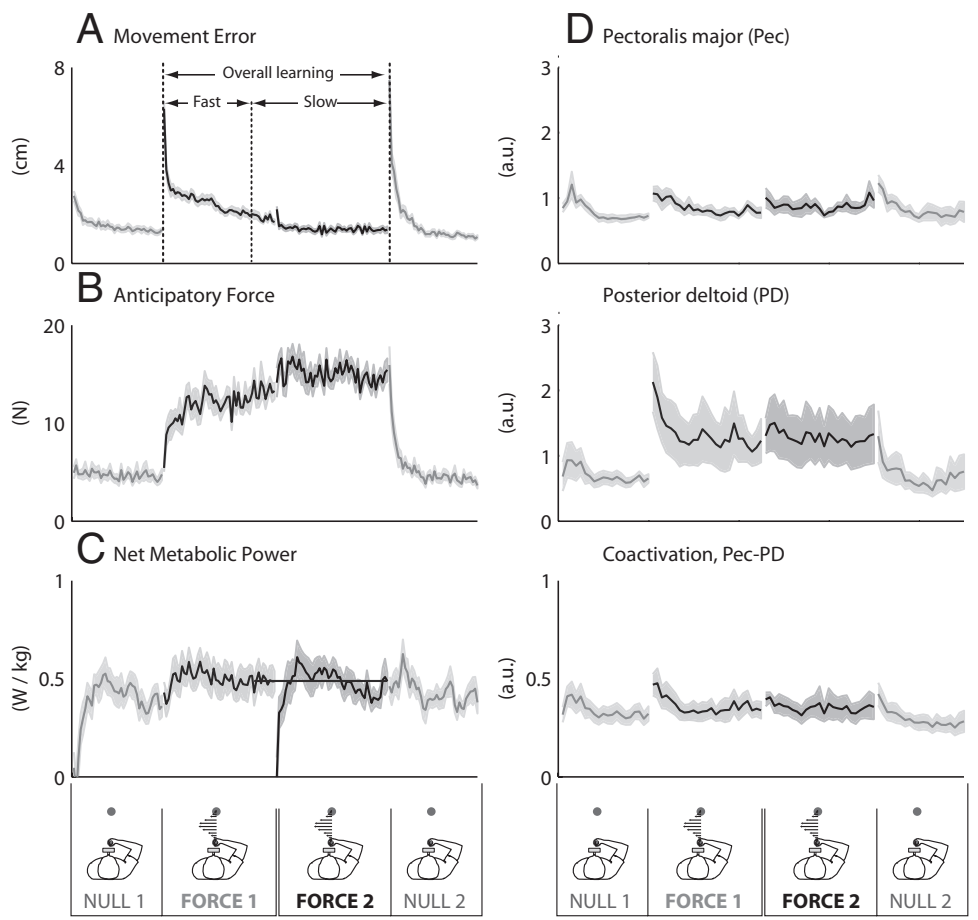

Figure 3. Time courses of movement error $(\boldsymbol{A})$, anticipatory force $(\boldsymbol{B})$, net metabolic power $(\boldsymbol{C})$, and RMS EMG and RMS coactivation $(\boldsymbol{D})$ by batches throughout the protocol. Lines are group means, and shaded areas depict \pm SEM. The dotted vertical lines in $\boldsymbol{A}$ outline that the overall learning period spans from early Force 1 to late Force 2, that fast learning occurs from early Force 1 to late Force 1 , and that slow learning occurs from late Force 1 to late Force 2. The dark gray horizontal thin line in net metabolic power $(\boldsymbol{C})$ represents the average for the last $2 \mathrm{~min}$ of Force 1 . This highlights that net metabolic power output during late Force 2 was less than during late Force 1 . Muscles and muscle pairs had similar time courses so only the shoulder muscle pair (pectoralis major and posterior deltoid) is shown. $N=7$ for EMG data and $N=15$ for all other measures. EMG and coactivation data were normalized by task to late Null 1 and are reported as arbitrary units (a.u.).

To quantify the initial effects of the novel dynamics of the curl force field, we examined the transition from late Null 1 to early Force 1. We expected large movement errors and small magnitudes of anticipatory force at early Force 1 compared with late Null 1 . We also expected greater net metabolic power for early Force 1 compared with late Null 1 , which would establish that initial reaching movements in the curl force field required greater metabolic expenditure. To examine our first hypothesis that metabolic power would decrease during motor learning, we compared movement error, anticipatory force, and net metabolic power for early Force 1 versus late Force 2, which spanned the entire learning period. A reduction in movement error and an increase in anticipatory force would indicate that subjects learned the novel dynamics, and a corresponding reduction in metabolic power would indicate that the nervous system was attempting to reduce metabolic expenditure during motor learning. To examine our second hypothesis that muscle coactivation would parallel the decrease in metabolic power, we compared RMS EMG and RMS coactivation for early Force 1 versus late Force 2. A corresponding decrease in RMS EMG and RMS coactivation would suggest that muscle activity and/or coactivation paralleled, and could explain the reduction of metabolic power during motor learning. We also compared all metrics for late Force 2 versus early Null 2 to quantify after-effects, another indicator that the dynamics were learned.

Fast motor learning comparisons. Because we expected movement patterns to change substantially during initial learning, we compared all variables for early Force 1 versus late Force 1. We refer to early Force 1 to late Force 1 as fast motor learning.

Slow motor learning comparisons. Even when movement patterns are just being fine-tuned, the CNS could still be attempting to reduce metabolic expenditure and learning to move more efficiently. To determine whether our data supported this idea, we compared movement error and anticipatory force for late Force 1 versus late Force 2 to assess whether the novel dynamics were still being learned. We refer to late Force 1 to late
Force 2 as slow motor learning. We then compared net metabolic power for late Force 1 versus late Force 2 to determine whether metabolic power was being reduced. Last, we compared RMS EMG and RMS coactivation for late Force 1 versus late Force 2 to examine again whether muscle activity and/or coactivation paralleled and could explain reductions in metabolic power.

Statistics. To assess statistical significance in motor learning, we used a repeated-measures ANOVA on all metrics to determine whether there was a main effect of time (i.e., early and late within each block). We then performed paired $t$ tests on all metrics for the following planned comparisons: (1) early Force 1 versus late Force 2 (overall motor learning), (2) early Force 1 versus late Force 1 (fast motor learning), and (3) late Force 1 versus late Force 2 (slow motor learning). Because we expected movement error to decrease with motor learning and anticipatory force to increase with motor learning, we used one-tailed paired $t$ tests for movement error and anticipatory force. We used two-tailed paired $t$ tests for metabolic power and EMG data. To determine whether there were significant differences in the transitions between blocks, we also performed paired $t$ tests on all metrics for the following planned comparisons: (1) late Null 1 versus early Force 1 , and (2) late Force 2 versus early Null 2 . The level of significance was set at $\alpha=0.05$. Exact $p$ values are reported for values greater than $p=$ 0.0001 .

\section{Results}

\section{Overview}

We first present results related specifically to overall motor learning, which spans the entire learning period from early Force 1 to late Force 2. We then present results specific to fast motor learning, from early Force 1 to late Force 1, when movements were changing substantially. Last, we present results from the slow motor learning period, from late Force 1 to late Force 2, when movements were being fine-tuned.

The time course of movement error, anticipatory force, muscle activity, muscle coactivation, and net metabolic power reveal the trends for each of these metrics during the different blocks of the entire protocol (Fig. 3). Throughout the protocol and across subjects, movement times were similar, $449 \pm 10 \mathrm{~ms}$ (mean \pm SEM). In Null 1, all metrics quickly stabilized around baseline levels (late Null 1). Initial movements in the curl force field had large movement errors, increased muscle activity, increased muscle coactivation, and low anticipatory forces (early Force 1). Subjects rapidly reduced movement error, muscle activity, and muscle coactivation and rapidly increased anticipatory force as learning progressed. From late Force 1 to late Force 2, further improvements occurred but were small in magnitude, indicating movements were stabilizing and being fine-tuned. When the curl force field was removed (early Null 2), there was a large movement error after-effect and subjects continued to produce anticipatory force, expecting to encounter the curl force field. These data indicate that subjects had learned the novel dynamics of the curl force field. Subjects then quickly decreased movement error, anticipatory force, muscle activity, muscle coactivation, and net metabolic power in Null 2. In each block, net metabolic power decreased after reaching an initial peak early in the block. 
Repeated-measures ANOVA indicated that time (i.e., early and late of each block) had a main effect on movement error, anticipatory force, and net metabolic power (all $p$ values $<0.0001$ ). Time also had a main effect on the biceps brachii ( $p=$ $0.0189)$, brachioradialis $(p=0.0074)$, triceps lateral head ( $p=0.0221)$, pectoralis major $(p=0.0006)$, posterior deltoid ( $p=0.0016)$, but not for the triceps long head ( $p=0.2066)$. Time had a main effect on all of the coactivation muscle pairs, the biceps brachii-triceps long head pair ( $p=$ $0.0391)$, brachioradialis-triceps lateral head pair ( $p=0.0206$ ), and pectoralis major-posterior deltoid pair $(p=$ 0.0001).

\section{Overall motor learning}

Movement traces and muscle

activity profiles

During early Force 1, upon initial exposure to the curl force field, movement paths had large perpendicular errors compared with the relatively straight line path at late Force 2 (Fig. 4A). Peak $y$-velocity and anticipatory force were also greater by the end of learning (Fig. 4B,C). Muscle activity patterns revealed that extensor muscles initiated outward movements and flexor muscles decelerated the arm. During early Force 1, subjects typically used greater muscle activity and coactivation in response to the novel curl force field but learned to decrease muscle activity and coactivation by late Force 2 (Fig. 4D)

\section{Movement error}

Group-averaged movement error during early Force 1 was $8.78 \pm 0.51 \mathrm{~cm}$ (Fig. $5 A$ ). By late Force 2, subjects reduced movement error to $1.36 \pm 0.11 \mathrm{~cm}(p<0.0001)$. When the curl force field was removed, errors increased significantly to $7.96 \pm 0.54 \mathrm{~cm}(p<0.0001)$ but then quickly decreased to $1.04 \pm 0.06 \mathrm{~cm}(p<0.0001)$.

\section{Anticipatory force}

Anticipatory forces at late Null 1, $4.53 \pm 0.52 \mathrm{~N}$, and early Force $1,5.43 \pm 0.40 \mathrm{~N}$, were small and not significantly different $(p=$ 0.1213 , Fig. $5 B$ ). While reaching in the curl force field, subjects increased anticipatory force by $182 \%$ from $5.43 \pm 0.40 \mathrm{~N}$ in early Force 1 to $15.29 \pm 0.95 \mathrm{~N}$ by late Force $2(p<0.0001)$. When the curl force field was removed at early Null 2, subjects initially continued to generate anticipatory forces, $15.92 \pm 1.91 \mathrm{~N}$, but quickly reduced anticipatory forces to $3.91 \pm 0.29 \mathrm{~N}$ by late Null $2(p<0.0001)$.

\section{Metabolic power}

The average resting baseline metabolic power was $1.29 \pm 0.04$ $\mathrm{W} / \mathrm{kg}$. Net metabolic power consumption increased by $42 \%$ from $0.38 \pm 0.05 \mathrm{~W} / \mathrm{kg}$ for late Null 1 to $0.54 \pm 0.06 \mathrm{~W} / \mathrm{kg}$ for early Force $1(p=0.0008)$, indicating that reaching in the curl force field required significantly greater metabolic power. Net metabolic power then decreased by $20 \%$ from $0.54 \pm 0.06 \mathrm{~W} / \mathrm{kg}$ for early Force 1 to $0.43 \pm 0.05 \mathrm{~W} / \mathrm{kg}$ for late Force $2(p=0.0183$, Fig. $5 C$ ), indicating that subjects reduced metabolic cost by 0.11 $\mathrm{W} / \mathrm{kg}\left(\sim 0.32 \mathrm{ml}\right.$ of $\left.\mathrm{O}_{2} / \mathrm{kg} / \mathrm{min}\right)$ during overall motor learning.

\section{Muscle activity and coactivation}

During the outward movements for which we recorded EMG data, significant reductions in muscle activity of the posterior deltoid ( $p=0.0098)$, biceps brachii $(p=0.0242)$, and brachioradialis ( $p=0.0329$ ) occurred over the span of the entire learning period, from early Force 1 to late Force 2 (Fig. 5D). Additionally, overall motor learning also corresponded with significant reductions in coactivation of the pectoralis major-posterior deltoid pair $(p=0.0327)$ and the biceps brachii-triceps long head pair $(p=0.0480)$.

\section{Fast motor learning}

During fast motor learning, from early Force 1 to late Force 1, movement error decreased from $8.78 \pm 0.51$ to $1.71 \pm 0.09 \mathrm{~cm}$ $(p<0.0001$, Fig. $5 A)$ and anticipatory force increased from $5.43 \pm 0.40$ to $13.19 \pm 0.76 \mathrm{~N}(p<0.0001$, Fig. $5 B)$. During that time, net metabolic power decreased by $8 \%$, from $0.54 \pm 0.06$ $\mathrm{W} / \mathrm{kg}$ at early Force 1 to $0.50 \pm 0.05 \mathrm{~W} / \mathrm{kg}$ at late Force $1(p=$ 0.1822 , Fig. $5 C$ ). Additionally, there were also significant reductions in muscle activity for the pectoralis major ( $p=$ $0.0190)$, posterior deltoid ( $p=0.0122$ ), and biceps brachii 
A Movement Error

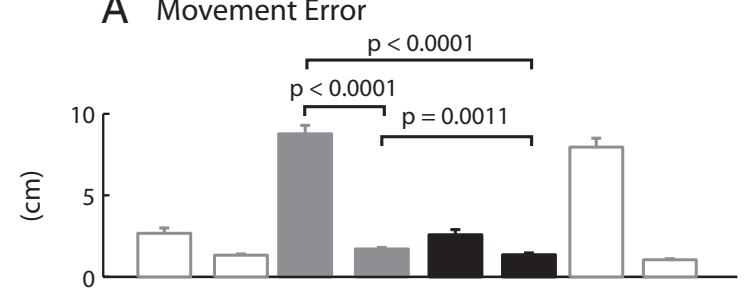

B Anticipatory Force

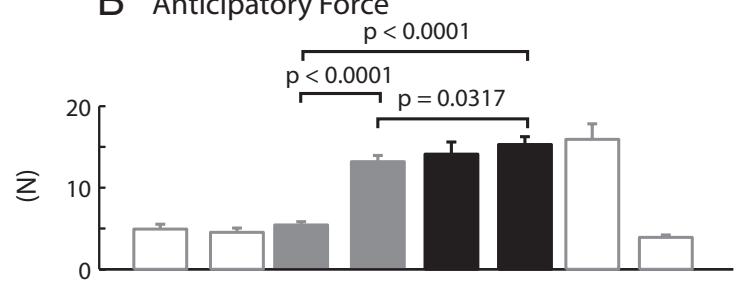

C Net Metabolic Power

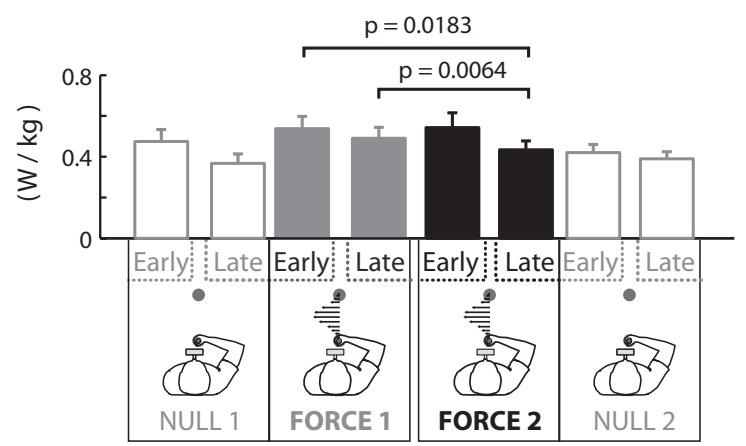

D Pectoralis major (Pec)
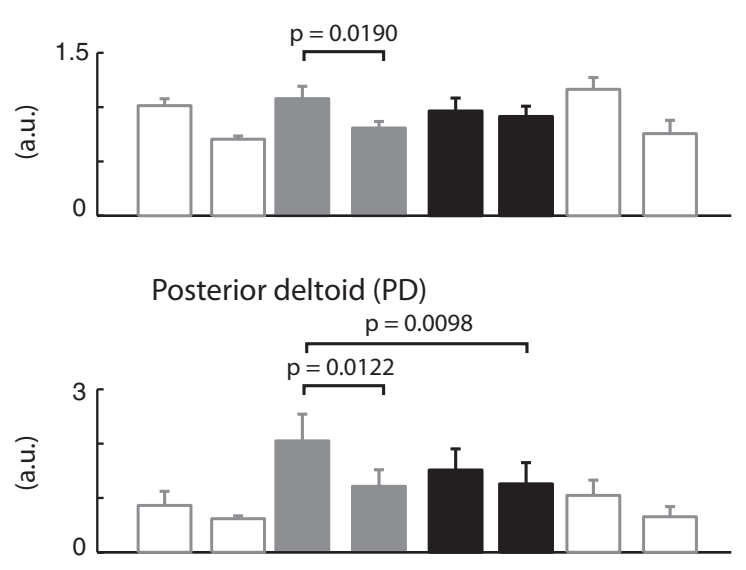

Coactivation, Pec-PD

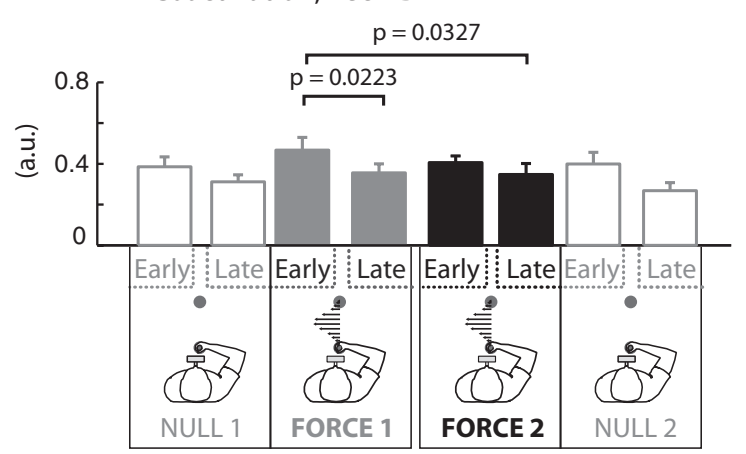

Figure 5. Group averaged data for movement error $(\boldsymbol{A})$, anticipatory force $(\boldsymbol{B})$, net metabolic power (C), and RMS EMG and RMS coactivation $(\boldsymbol{D})$ during overall, fast, and slow motor learning. During overall motor learning from early Force 1 to late Force 2, movement error, net metabolic power, RMS EMG of the posterior deltoid, and RMS coactivation of the pectoralis major-posterior deltoid pair decreased significantly and anticipatory force also increased significantly. During fast motor learning, early Force 1 to late Force 1 , all metrics except net metabolic power had significant changes. During slow motor learning, late Force 1 to late Force 2, net metabolic power decreased significantly as movement error and anticipatory force also improved significantly, though small in amplitude. Muscles and muscle pairs had similar trends so only the shoulder muscle pair (pectoralis major and posterior deltoid) is shown. Error bars indicate SEM. $P$ values are for paired $t$ tests of planned comparisons. $N=7$ for EMG data and $N=15$ for all other measures. EMG and coactivation data were normalized by task to late Null 1 and are reported as arbitrary units (a.u.).

( $p=0.0230)$ and for the pectoralis major-posterior deltoid coactivation pair $(p=0.0223$; Fig. $5 D)$.

\section{Slow motor learning}

Movement traces and muscle activity profiles

Movement paths in late Force 1 and late Force 2 were typically relatively straight line paths and similar to baseline, late Null 1 (Fig. 6A). Y-velocity profiles were also similar, having comparable magnitudes and bell-shaped profiles (Fig. 6B). However, peak anticipatory force of late Force 2 was greater than late Force 1 (Fig. 6C). Muscle activity and coactivation profiles at late Force 1 and late Force 2 were similar to late Null 1 (Fig. 6D).

\section{All metrics}

Additional improvements in movement patterns during the latter half of learning were small in magnitude and demonstrate fine-tuning of movements. Reductions in movement error were significant $(0.35 \pm 0.09 \mathrm{~cm} ; p=0.0011)$ and increases in anticipatory force were also significant $(2.10 \pm 1.04 \mathrm{~N} ; p=0.0317)$ from late Force 1 to late Force 2 (Fig. $5 A, B$ ). Interestingly, net metabolic power decreased by $14 \%$ from $0.50 \pm 0.05 \mathrm{~W} / \mathrm{kg}$ for late Force 1 to $0.43 \pm 0.05 \mathrm{~W} / \mathrm{kg}$ for late Force 2 ( $p=0.0064$, Fig. $5 C)$. This decrease was evident in 13 of the 15 subjects, indicating that this decrease in net metabolic power was consistent among subjects (Fig. 7). However, there were no significant differences in muscle activity from late Force 1 to late Force 2 for the biceps brachii $(p=0.9297)$, brachioradialis $(p=0.7900)$, pectoralis major $(p=0.2593)$, posterior deltoid $(p=0.6664)$, triceps lateral head $(p=0.5210)$, and triceps long head $(p=0.3476)$. There were also no significant differences in coactivation, biceps brachii-triceps long head ( $p=0.6459)$, brachioradialis-triceps lateral head ( $p=0.7213)$, and pectoralis major-posterior deltoid $(p=0.6226)$. Even though changes in muscle activity and muscle coactivation were small and not statistically significant from late Force 1 to late Force 2, the significant improvements in movement error and anticipatory force suggest that motor learning was still ongoing and being fine-tuned. The concomitant reduction in metabolic power during this period indicates that metabolic power was reduced during motor learning.

\section{Discussion}

This is the first demonstration that net metabolic power decreases with motor learning (early Force 1 to late Force 2) in an arm reaching task, supporting our first hypothesis that metabolic power output would decrease as the novel dynamics were learned. Interestingly, metabolic power reductions continued to occur late in motor learning (late Force 1 to late Force 2) whereas muscle activity decreases were only detected during initial motor learning (early Force 1 to late Force 1). The differing time scales 
and disproportionate changes in metabolic power and muscle activity reductions do not support our second hypothesis that muscle activity and coactivation would parallel the decrease in metabolic power.

The most intriguing finding was that net metabolic power continued to decrease consistently among subjects even when movements were being fine-tuned and EMG patterns had stabilized. Initially, during fast motor learning when there were significant changes in movement error, anticipatory force, muscle activity, and muscle coactivation, the reduction in net metabolic power was not statistically significant $(\sim 0.04 \mathrm{~W} / \mathrm{kg} ; p=$ 0.1822 ). However, during slow motor learning, when there were only small but significant improvements in movement error and anticipatory force, metabolic power decreased further $(\sim 0.07 \mathrm{~W} / \mathrm{kg}$; $p=0.0064)$. In contrast to metabolic power, muscle activity and coactivation were not observed to decrease. Therefore, observed changes in muscle activity were not proportional to changes in metabolic power and did not parallel the reduction of metabolic power during motor learning. This challenges the widely held assumption that muscle activity entirely explains changes in metabolic cost. Other mechanisms in addition to the reduction of muscle activity and coactivation appear to underlie the decrease in metabolic cost later in motor learning.

Our results provide the first evidence of actual metabolic reduction during motor learning and during reaching. Previously, decreases in muscle coactivation and stiffness were used to suggest that metabolic cost must also decrease (Thoroughman and Shadmehr, 1999; Franklin et al., 2003, 2004, 2008; Darainy and Ostry, 2008). Our muscle activity and coactivation data also decrease rapidly with learning, within 50-100 trials, and then settled around an asymptotic level (Thoroughman and Shadmehr, 1999; Franklin et al., 2003; Darainy and Ostry, 2008). However, because we measured actual metabolic cost, we found that the rapid decreases in muscle activity and coactivation did not correspond with rapid decreases in metabolic power. Rather, greater metabolic power reductions occurred when muscle activity and coactivation had reached asymptotic levels.

We have demonstrated that the metabolic cost of reaching is measurable and is not insignificant. Although arm reaching seems metabolically inexpensive, the gross metabolic power expenditure for seated reaching during our protocol was $\sim 1.68 \mathrm{~W} / \mathrm{kg}$. For reference, the gross metabolic power during standing is $\sim 1.5 \mathrm{~W} / \mathrm{kg}$ (Grabowski et al., 2005; Houdijk et al., 2009; Snyder and Farley, 2011). The net metabolic power for normal walking (at $1.25 \mathrm{~m} / \mathrm{s}$ ) is $\sim 3 \mathrm{~W} / \mathrm{kg}$ (Gottschall and Kram, 2003; Grabowski et al., 2005; Collins et al., 2009; Farris and Sawicki, 2011). Therefore, reaching itself is equivalent to $\sim 13 \%$ of the cost of normal walking and reaching in the curl force field increased to $\sim 18 \%$ of the cost of normal walking.
Consequently, there is an incentive to reduce metabolic power during reaching.

Indeed, the CNS is sensitive to small differences in metabolic power. Over the entire learning period, net metabolic power decreased by $\sim 3.5 \%$ the cost of normal walking. During slow motor learning, net metabolic power decreased by $\sim 2 \%$ the cost of normal walking, which may reflect the sensitivity of the CNS to metabolic cost. Additionally, metabolic power decreased in $77 \%$ of the subjects from early to late within a block [ 46 reductions/(15 subjects ${ }^{\star} 4$ blocks)], indicating a general tendency to decrease metabolic cost. Even when subjects were just reaching from early to late Null 1, net metabolic power decreased by $\sim 0.10 \mathrm{~W} / \mathrm{kg}$ $(p=0.0043)$. The CNS seems to reduce metabolic expenditure in general during learning and movement.

Practice over multiple sessions can reduce metabolic expenditure. When learning to arm cycle (Sparrow et al., 2005; Galna and Sparrow, 2006), leg cycle (Lay et al., 2005), row (Lay et al., 2002), and walk with ankle-foot orthoses (Sawicki and Ferris, 2008), metabolic expenditure decreased with repeated practice. Similarly, subjects can learn to walk backwards at faster speeds with 


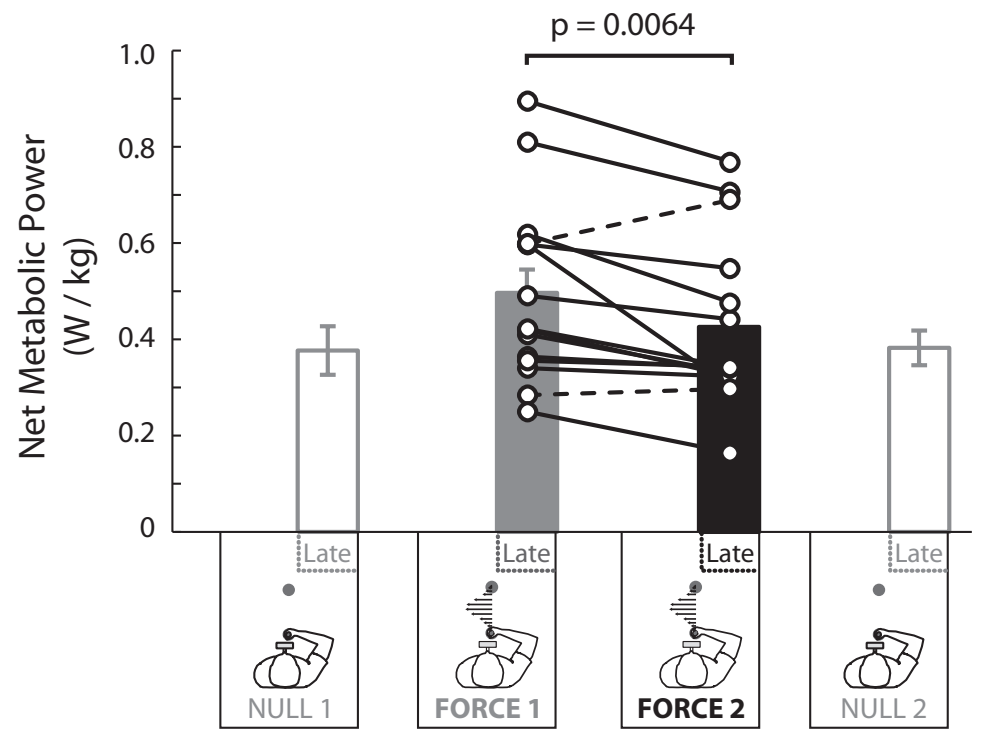

Figure 7. Reduction in net metabolic power consumption during slow motor learning in all individual subjects. When movements and muscle activity were being fine-tuned, group averaged net metabolic power consumption still decreased significantly by $14 \%$ from late Force 1 to late Force $2(0.50 \pm 0.05 \mathrm{~W} / \mathrm{kg}$ vs $0.43 \pm 0.05 \mathrm{~W} / \mathrm{kg})$. This metabolic reduction was consistent among subjects. Thirteen of 15 subjects (solid lines) reduced net metabolic power from late Force 1 to late Force 2, whereas only two subjects (dashed lines) increased net metabolic power. Net metabolic power of late Null 1 and late Null 2 were not significantly different $(p=0.7726)$. Error bars indicate SEM. $P$ values are paired $t$ tests of planned comparisons.

the same metabolic rate, indicating improved metabolic economy (Childs et al., 2002). Interestingly, significant reductions in metabolic expenditure occurred later, when coordination patterns had stabilized (Childs et al., 2002; Sparrow et al., 2005; Galna and Sparrow, 2006). We also observed further metabolic reductions when reaching movements were being fine-tuned. However, our metabolic reductions occurred within a single session of only $\sim 20 \mathrm{~min}$, in contrast to multiple sessions.

Other studies have attempted to determine whether reaching trajectories are chosen to minimize metabolic cost, but have not unequivocally established a causal link. Training subjects to use nonpreferred trajectories is difficult and hinders the ability to experimentally test whether movement trajectories are chosen to minimize metabolic cost (Alexander, 1997). In a recent study, subjects continued to move in a relatively straight line path to the target, even though it required greater end-point work than a curved movement path that was designed to be the minimum end-point work path (Kistemaker et al., 2010). They concluded that the CNS does not minimize metabolic cost. Without measuring actual metabolic expenditure, it is unknown whether their curved movement path actually required less metabolic power. Our data also demonstrate that subjects could exert greater end-point force $(\sim 2 \mathrm{~N})$ while consuming less metabolic power.

The fact that metabolic power reductions were not proportional to observed changes in muscle activity suggests that other mechanisms may also underlie metabolic reductions. Our results indicate that when learning novel dynamics, movement patterns adapted rapidly, corresponding with rapid changes in muscle activity. With further learning, movement patterns were finetuned while observed muscle activity amplitudes were asymptotic, suggesting that other features of muscle activity were being fine-tuned. During this fine-tuning, standard metabolic measurements (i.e., average of late Force 1, average of late Force 2) revealed a significant metabolic reduction. Fine-tuning of arm muscle activity features when amplitudes were asymptotic may underlie this reduction in metabolic power. Further, decreased activity of other muscles (i.e., postural muscles) may also contribute to the metabolic reduction. Another possibility is that improved neural efficiency, such as using less brain activity and/or optimizing motor unit recruitment, could reduce metabolic cost. Efficient neuronal signaling in the brain has been shown to correspond with energy minimization (Attwell and Laughlin, 2001; Hasenstaub et al., 2010). Information processing and neuronal signaling patterns also consume a large portion of the total energy used by the brain (Attwell and Laughlin, 2001; Magistretti, 2009). This suggests that ultimately, efficient movements involve both efficient biomechanics and efficient neural processes (i.e., muscle activation and thinking).

A limitation of this study is that the precise time delays between changes in movement and expired gases and between changes in expired gases and measured metabolic rate are unknown. For this reason, we did not include the first $2 \mathrm{~min}$ in a block when estimating metabolic power early within the block. Further, these estimates may not have captured the actual peak initial cost, particularly for early Force 1 . We used 2 min when averaging metabolic power because it was less variable than $1 \mathrm{~min}$ but also short enough to detect differences early and late within a block. Our conclusions would have been the same whether we used 1 or 3 min, while durations longer than 4 min did not detect significant reductions. Nevertheless, the unknown time delays may affect the interpretation of the time course of metabolic power in relation to the time courses of the other variables. Another limitation is that surface electromyography of the selected arm muscles may not have been able to detect changes in muscle activity during slow motor learning. In-dwelling electromyography, measurement of other muscles, and other analyses of muscle activity may have been able to detect differences that surface electromyography and amplitude metrics did not.

Measuring actual metabolic cost may be a useful tool for future motor learning studies. We have shown that metabolic power can be measured continuously and interpreted with respect to changes in movement patterns. Additionally, small differences in metabolic power can be measured consistently. Importantly, these results highlight that end-point force, endpoint work, and/or electromyography do not necessarily reflect changes in metabolic cost. Thus, actual metabolic cost can and should be measured when investigating hypotheses related to metabolic cost. An important question for future research is how does actual metabolic cost trade-off with task performance and motor learning in clinical populations. Future studies could also examine the relationships among brain activity, metabolic cost, and motor learning.

In summary, we have demonstrated that (1) net metabolic power decreased during overall motor learning, and (2) reductions in metabolic power occurred later during motor learning, when movements were being fine-tuned. These findings suggest that during motor learning, the CNS reduces metabolic expenditure and may also optimize neural activity to become more efficient. 


\section{References}

Alexander RM (1997) A minimum energy cost hypothesis for human arm trajectories. Biol Cybern 76:97-105.

Alexander R (2002) Energetics and optimization of human walking and running: The 2000 Raymond Pearl Memorial Lecture. Am J Hum Biol 14:641-648.

Arellano CJ, Kram R (2011) The effects of step width and arm swing on energetic cost and lateral balance during running. J Biomech 44: 1291-1295.

Attwell D, Laughlin SB (2001) An energy budget for signaling in the grey matter of the brain. J Cereb Blood Flow Metab 21:1133-1145.

Brockway JM (1987) Derivation of formulae used to calculate energyexpenditure in man. Hum Nutr Clin Nutr 41:463-471.

Brooks GA, Fahey TD, White TP (1996) Exercise physiology: human bioenergetics and its applications, Ed 2. Mountain View, CA: Mayfield.

Burden A (2010) How should we normalize electromyograms obtained from healthy participants? What we have learned from over 25 years of research. J Electromyogr Kinesiol 20:1023-1035.

Cavanagh PR, Williams KR (1982) The effect of stride length variation on oxygen-uptake during distance running. Med Sci Sports Exerc 14:30-35.

Childs JD, Gantt C, Higgins D, Papazis JA, Franklin R, Metzler T, Underwood FB (2002) The effect of repeated bouts of backward walking on physiologic efficiency. J Strength Cond Res 16:451-455.

Collins SH, Adamczyk PG, Kuo AD (2009) Dynamic arm swinging in human walking. Proc R Soc Lond B Biol Sci 276:3679-3688.

Cotes JE, Meade F (1960) The energy expenditure and mechanical energy demand in walking. Ergonomics 3:97-119.

Cram JR, Kasman GS (1998) Introduction to surface electromyography. Gaithersburg, MD: Aspen.

Darainy M, Ostry DJ (2008) Muscle cocontraction following dynamics learning. Exp Brain Res 190:153-163.

Donelan JM, Kram R, Kuo AD (2001) Mechanical and metabolic determinants of the preferred step width in human walking. Proc R Soc Lond B Biol Sci 268:1985-1992.

Donelan JM, Kram R, Kuo AD (2002) Mechanical work for step-to-step transitions is a major determinant of the metabolic cost of human walking. J Exp Biol 205:3717-3727.

Emken JL, Benitez R, Sideris A, Bobrow JE, Reinkensmeyer DJ (2007) Motor adaptation as a greedy optimization of error and effort. J Neurophysiol 97:3997-4006.

Farris DJ, Sawicki GS (2012) The mechanics and energetics of human walking and running: a joint level perspective. J R Soc Interface 9:110-118.

Franklin DW, Osu R, Burdet E, Kawato M, Milner TE (2003) Adaptation to stable and unstable dynamics achieved by combined impedance control and inverse dynamics model. J Neurophysiol 90:3270-3282.

Franklin DW, So U, Kawato M, Milner TE (2004) Impedance control balances stability with metabolically costly muscle activation. J Neurophysiol 92:3097-3105.

Franklin DW, Burdet E, Tee KP, Osu R, Chew CM, Milner TE, Kawato M (2008) CNS learns stable, accurate, and efficient movements using a simple algorithm. J Neurosci 28:11165-11173.

Galna B, Sparrow WA (2006) Learning to minimize energy costs and maximize mechanical work in a bimanual coordination task. J Mot Behav $38: 411-422$.
Gottschall JS, Kram R (2003) Energy cost and muscular activity required for propulsion during walking. J Appl Physiol 94:1766-1772.

Grabowski A, Farley CT, Kram R (2005) Independent metabolic costs of supporting body weight and accelerating body mass during walking. J Appl Physiol 98:579-583.

Gribble PL, Mullin LI, Cothros N, Mattar A (2003) Role of cocontraction in arm movement accuracy. J Neurophysiol 89:2396-2405.

Hasenstaub A, Otte S, Callaway E, Sejnowski TJ (2010) Metabolic cost as a unifying principle governing neuronal biophysics. Proc Natl Acad Sci U S A 107:12329-12334.

Holt KG, Hamill J, Andres RO (1991) Predicting the minimal energy costs of human walking. Med Sci Sports Exerc 23:491-498.

Houdijk H, Fickert R, van Velzen J, van Bennekom C (2009) The energy cost for balance control during upright standing. Gait and Posture 30:150-154.

Izawa J, Rane T, Donchin O, Shadmehr R (2008) Motor adaptation as a process of reoptimization. J Neurosci 28:2883-2891.

Kistemaker DA, Wong JD, Gribble PL (2010) The central nervous system does not minimize energy cost in arm movements. J Neurophysiol 104:2985-2994.

Kuo AD (2001) A simple model of bipedal walking predicts the preferred speed-step length relationship. J Biomech Eng 123:264-269.

Lay BS, Sparrow WA, Hughes KM, O'Dwyer NJ (2002) Practice effects on coordination and control, metabolic energy expenditure, and muscle activation. Hum Mov Sci 21:807-830.

Lay BS, Sparrow WA, O'Dwyer NJ (2005) The metabolic and cognitive energy costs of stabilising a high-energy interlimb coordination task. Hum Mov Sci 24:833-848.

Magistretti PJ (2009) Low-cost travel in neurons. Science 325:1349-1351.

Margaria R (1976) Biomechanics and energetics of muscular exercise. Oxford: Clarendon.

Nelson WL (1983) Physical principles for economies of skilled movements. Biological Cybernetics 46:135-147.

Ralston HJ (1958) Energy-speed relation and optimal speed during level walking. Int Z Angew Physiol 17:277-283.

Sawicki GS, Ferris DP (2008) Mechanics and energetics of level walking with powered ankle exoskeletons. J Exp Biol 211:1402-1413.

Shadmehr R, Mussa-Ivaldi FA (1994) Adaptive representation of dynamics during learning of a motor task. J Neurosci 14:3208-3224.

Snyder KL, Farley CT (2011) Energetically optimal stride frequency in running: the effects of incline and decline. J Exp Biol 214:2089-2095.

Sparrow WA, Parker S, Lay B, Wengier M (2005) Aging effects on the metabolic and cognitive energy cost of interlimb coordination. J Gerontol A Biol Sci Med Sci 60:312-319.

Thoroughman KA, Shadmehr R (1999) Electromyographic correlates of learning an internal model of reaching movements. J Neurosci 19:8573-8588.

Todorov E, Jordan MI (2002) Optimal feedback control as a theory of motor coordination. Nat Neurosci 5:1226-1235.

Yang JF, Winter DA (1984) Electromyographic amplitude normalization methods: improving their sensitivity as diagnostic tools in gait analysis. Archives of Physical Medicine and Rehabilitation 65:517-521.

Zarrugh MY, Radcliffe CW (1978) Predicting metabolic cost of level walking. Eur J Appl Physiol Occup Physiol 38:215-223.

Zarrugh MY, Todd FN, Ralston HJ (1974) Optimization of energy expenditure during level walking. Eur J Appl Physiol Occup Physiol 33:293-306. 